\title{
Comprehensive Assessment of Wearable Radiating Textile Sensorics \\ ${ }^{1}$ Shashikant Patil, ${ }^{2}$ Pravin Wararkar, ${ }^{3}$ Mayank Kothari \\ ${ }^{1,2,3}$ Department of Electronics \& Telecommunication Engineering, SVKM's NMIMS, MPSTME Shirpur, Dhule Email: sspatil999@gmail.com,pwararkar@gmail.com, mayankkothari28@gmail.com
}

Received: 09 ${ }^{\text {th }}$ July 2018, Accepted: $14^{\text {th }}$ August 2018, Published: 31 ${ }^{\text {st }}$ August 2018

\begin{abstract}
Latest E-textile technologies needs wireless medium and channel as well as appreciable coverage area with small size and robust structure. The sensors embedded inside textile material must be insensitive to noise and comfortable to wear. This review paper summarizes and addressing the Ultra High Frequency Radio Frequency Identification (UHF RFID) tags equipped all the body worn textile sensors. UHF RFID tags are the type of sensors which allow usage of wireless communication up to a certain distance. This is an attempt to provide new insights in wearable devices with aid of Electronics augmented with Textile Technology for better comfort and reliability.
\end{abstract}

Keywords: E-Textile, RFID, Antenna Sensor, Backscattered, PIFA

\section{Introduction}

Present generation is very much interested in body worn communication system and textile antennas. These antennas can be easily fitted in cloths. Cloths are integrated with RFID sensors. These RFID sensors are Radio Frequency Identification Sensors which helps in detection of motion from several feet away and does not require direct contact of device. They work on electromagnetic fields. When these RFID sensors are attached to textile material if gives generation to wireless wearable textile sensors. These wearable sensors have wide range of use in real world. Some of these uses will be discussed below [7].

\section{A. Textile Antenna Sensors for on-body:}

The need for developing body worn sensor is to be capable of recording the activities, body conditions, physiological parameters and user location form collected data. These body worn sensor finds its application in the field of military, rescue, medical or entertainment world. Depending upon the application the frequency range is decided.

Major parameters and points should be kept in mind while designing developing and deploying of textile Antennas:

1. Radiating Antenna sensor must be safe for human being and human body.

2. Sensors geometry and dimensions must be taking care of wearable comfort and properties.

3. Antennas efficiency should not be compromised. Thus to integrate antenna sensor in textile microchips are proposed. Fig depicts the design and geometry of the same [1].

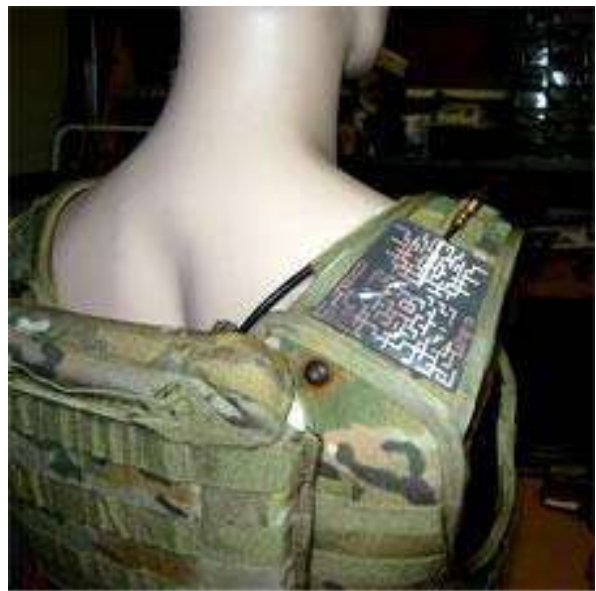

Figure 1: Direction Finding Antennas [8]. PIFA Antennas Based Sensors

PIFA antennas can be introduced in fabric to achieve few requirements. PIFA (Planar Inverted-F Antenna) reduces the required space. It has good SAR (Specific Absorption Rate) properties. SAR measures how transmitted RF energy is absorbed by human tissues. PIFA has low profile and has an omnidirectional pattern [9].

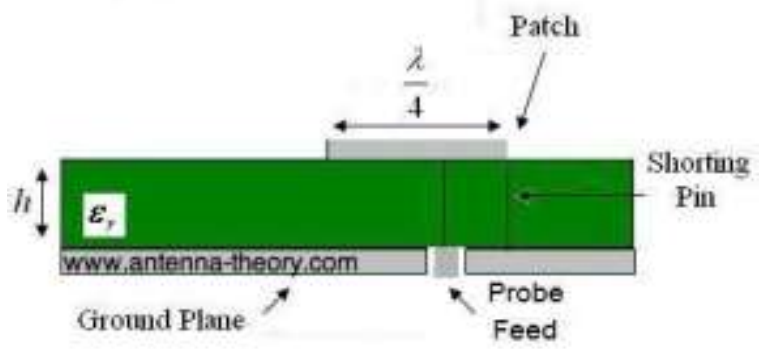

Figure 2: The Planar Inverted-F Antenna (PIFA) [10].

Human Body and Wireless Link

Human body is directly or indirectly exposed to wearable antenna sensor. Due to such exposure, radiation efficiency is reduced. For In-body communication, the efficiency range of antennas can be below $1 \%$. But for off-body or on-body communication, the efficiency must be $10 \%$ to $40 \%$. Thus it is observed that properties of skin and tissue 
determine the penetration of electromagnetic field at microwave frequency.

\section{Sensors Integration in Textile Material}

Designing an E-textile using antenna sensors is one of the challenging task. Antenna sensors require conductive base to work properly. Thus conductive cloths are used for preparation of E-textile/wearable textile sensor. Such cloth must also pose good properties of cloth (low price, aesthetic, comfortable, wash ability, drivability etc). Only conductive threads are very costly. Thus mixture of conductive and nonconductive threads is used for preparation of cloths.
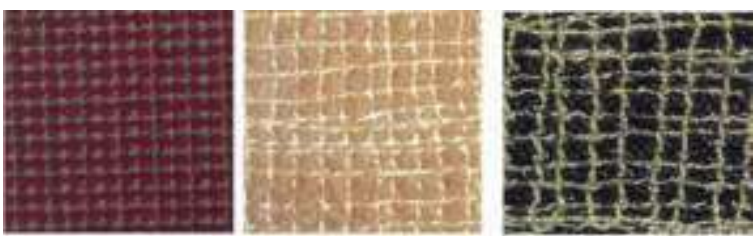

Figure 3: Samples of Conductive Threads Embroided in Felt, Wool and Denim

\section{B. Body-worn Antennas Sensor and E-Textile:}

Conductive textile device are formed by embroidering metal-coated polymer E-fabric onto our everyday garment. Programmable sewing machine is used for this automatic fabrication. In programmable sewing machine, yarns design by combining silver-coated pphnylene-2, 6-benzoisoxazole (PBO) or liquid crystal polymer (LCP) filament E-fibers are used. In addition to these fibers, textile antennas are embedded on polymer substrate. These polymer substrate are kind of paper but are more durable than paper. They won't get soaked in liquid and have hard surface. Such combination provides good RF performance, mechanical strength and flexibility. Such E-textile has as good RF performance that of copper [2].

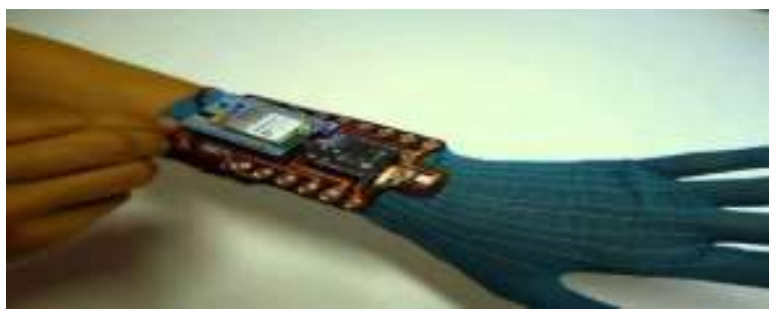

Figure 4: Example E-textile applications [11].

\section{Wireless Strain-Sensors with passive E-Textile UHF RFID System:}

RFID strain gauge sensors are the sensors which do not require any batteries or wires. Passive RFID systems do not have any internal power sources. They are powered by electromagnetic energy transmitted form RFID reader. They have two main components: tag's antenna and microchip or IC (integrated circuit). They do not need any maintenance and work lifelong.
Passive RFID tags use modulated scattering mechanism for establishing ultra-low-power radio communication over a distance of several meters. This is the means by which reader and the tag interact with each other using reciprocity theorem based on Maxwell equation.

This RFID enabled strain sensors are based on backscattered readout mechanism. EM optimization of coupled two-tag system in which one tag is sensitive and other is insensitive towards strain sensor is used for achieving backscattered power at reader antenna with distance ' $d$ ' from tags.

The sensors and there reference tags are based on dipole antennas with an embedded inductance matching loops. The reference tags are made up of non-stretchable copper plated polyester textile. The sensor on the other hand use stretchable and nonstretchable textile. EM coupling between antennas in sensor and reference tag is one of the main challenges in optimization of sensor. That changes the radiation properties and input impedance. To control this alteration, two-antenna system must be considered [3].

\section{Wireless Electronic Interface for Wearable Textile Sensors:}

A wearable resistive textile sensors, are provided with wireless electronic interface which meets the requirement and provide real time measurement. Electronic interfacing is the term used when different electronic devices are connected in order to move different information from one device to another.

Resistive textile sensors when integrated in clothes, provide bio-mechanical or physiological information. In wearable textile sensor, electronic interface acquires some basic features. They should have large resistance measurement range, should provide robust circuit to avoid noise. Must have simplified and smaller size and should consume less power with longer service time. ADC quantization error and reference resistance error are the two kind of error provides by Electronic Interface. The difference between actual analog value and quantized digital value is termed as Quantization error whereas the difference between real value of used and set value of is termed as reference resistance error. With the help of high precision nominal resistance these errors are minimized and that can be very near to set value. Such kind of Electronic Interface has been implemented in a plentar foot measurement system. Thus, Electronic Interface provides wireless transmission, large range of measurement robustness and real time measurement ability [4]. 


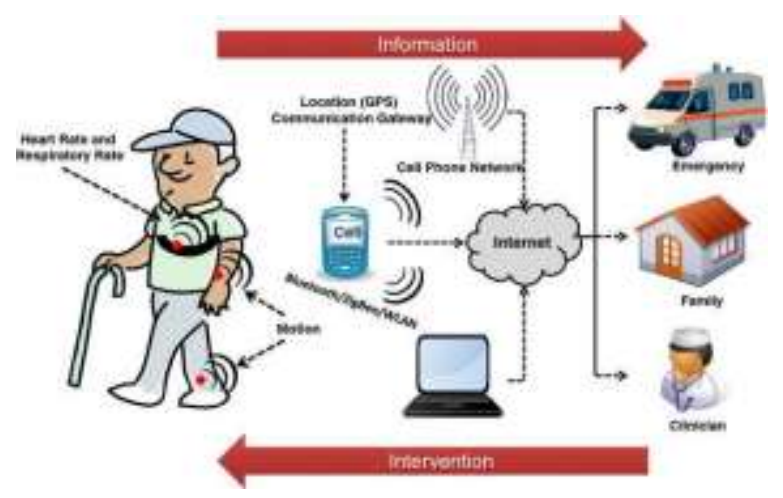

Figure 5: Working of On-body Sensor [12].

\section{E. E-textile Antenna with passive UHF RFID sensing:}

Wireless passive UHF RFID strain sensor tags work on backscattered mechanism. To check relation between backscattered strength and antenna elongation, two wirelessly different sensors are used with $1 \mathrm{~cm}$ and $3 \mathrm{~cm}$ stretchable section. They were characterized with minimal sensor read out distance and their response to backscattered strength to antenna elongation. The test result showed that backscattered strength has linear relationship with antenna elongation.

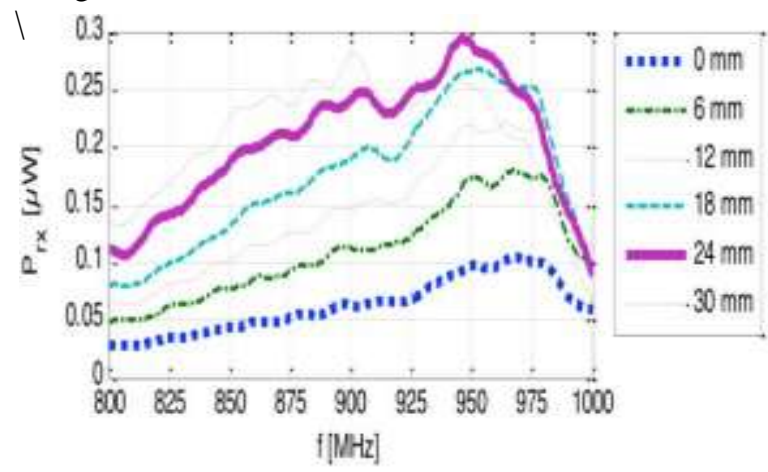

Figure 6: The Backscattered Signal Power from Tag A (Top) and Tag B (Bottom) for various Antenna Elongations [5].

The digitally modulated scattering based on radiofrequency indication tags provide long distance communication over distance of 25 meters in ultrahigh frequency (UHF) without any source. To study sensor tag antenna, T-matched dipole are used which uses antenna configuration in UHF RFID tags. These dipole antennas are linearly polarized along $\mathrm{x}$-axis and Omnidirectional radiation in YZ plane. Such test shows that swing of two highly conductive textile thread modifies the antenna properties. More over antenna elongation and backscattered strength have linear relationship. [6]

\section{Conclusion}

Passive RFID tags consist of antenna based sensing with minimal increase in complex and power consumption. Textile antenna with stretchable section using RFID strain sensor tag modifies the antenna properties. The electrical interconnection of two fabric are done using conductive yarn stitching.

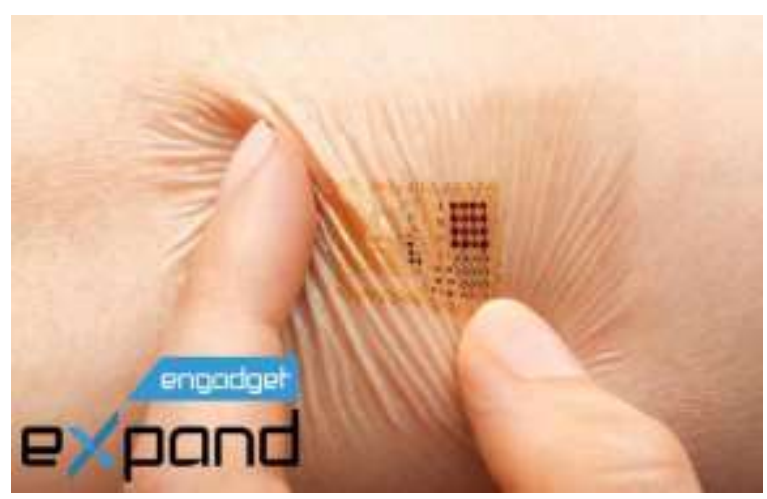

Figure 7: Future of Wearable Sensors [12]

E-textiles which can be embedded in body and can be considered as the future technology in healthcare management systems like having usage of such RFID tag sensors. Thus, this UHF RFID E-textile sensor are important part of life science and technology. These sensors along with sophisticated technologies can be used for data collection and data analytics of human body parameters and diagnosis of the variety of diseases. In future such sensoric systems will take care of Health diagnosis and analysis. In Future wearable textile sensors can be designed from the perspective of power consumption compact light weight body compatible and with faster switching actions and cater the services with increased coverage area along with decision making ability and self-healing systems which can be beneficial from societal aspects.

\section{References}

1. Branimir Ivsic, Dovor Bonefacic, Juraj Bartolic Department for wireless communication UniversityOf Zagreb, FERZagreb, Croatiae-mail branimir.ivsic@fer.her davor.bonefacic@fer.hr , juraj.bartolic@ fer.hr,’'Textile Antennas for On-body sensor", 978-1-4799-6117-7/15/\$31.00 @2015 IEEE

2. Asimina Kiourti and John L. Volakis Electro Science Laboratory, Department of Electrical and Computer Engineering The Ohio State University Columbus, OH 43212, USA kiourti.1@osu.edu, volakis.1@osu.edu 978-1-4799-7815-1/15/\$31.00 (2) 2015 IEEE

3. Xiaochen Chen, Leena Ukkonen, Member IEEE, and Toni Björninen, Member IEEE," Passive E- 
Helix Vol. 8(5): 3912- 3915

Textile UHF RFID based Wireless Strain Sensors with Integrated References",1530-437X (c) 2016 IEEE.

4. Lin Shu, Xiao Ming Tao*, MIEEE Institute of Textiles and Clothing The Hong Kong Polytechnic University Hong Kong*corresponding author, tctaoxm@polyu.edu.hk," A Wearable, Wireless Electronic Interface for Textile Sensors", 978-1-42445309-2/10/\$26.00 @2010 IEEE

5. Feiyuan Long1, Xiao Dong Zhang1, Toni Björninen2, Johanna Virkki2,Lauri Sydänheimo2, Yan-Cheong Chan1, Leena Ukkonen21 Shenzhen Research Institute / Department of Electronic Engineering, City University of Hong Kong, Hong Kong, China2 Department of Electronics and Communications Engineering, Tampere University of Technology, Tampere, Finland," Implementation and Wireless Readout of Passive UHF RFID Strain Sensor Tags based on Electro-Textile Antennas",

6. http://wirelesslifesciences.org/2014/11/mc10takes-the-guesswork-out-of-fitness-with-wearablesensors/

7. www.sheffield.ac.uk

8. http://www.megawave.com/pages/products.php

9. www.antenna-theory.com

10.http://www.antennatheory.com/antennas/patches/p ifa.php

11.http://www.keyglove.net/wp-

content/uploads/2010/10/xosc_glove.jpg

12.https://static-humacontent.springer.com/ esm/art\%3A10.1186\%2F1743-0003-9-

21/MediaObjects/12984_2011_353_MOESM1_ESM

.jpeg 